\title{
Pilot pulsar surveys with LOFAR
}

\author{
Thijs Coenen ${ }^{1}$ \\ on behalf of the LOFAR Pulsar Working Group \\ ${ }^{1}$ Anton Pannekoek Instituut, Universiteit van Amsterdam, \\ Postbus 94249, Amsterdam, The Netherlands \\ email: t.coenen@uva.nl
}

\begin{abstract}
We are performing two complementary pilot pulsar surveys as part of LOFAR commissioning. The LOFAR Pilot Pulsar Survey (LPPS) is a shallow all-sky survey using an incoherent combination of LOFAR stations. The LOFAR Tied-Array Survey (LOTAS) is a deeper pilot survey using 19 simultaneous tied-array beams. These will inform a forthcoming deep survey of the entire northern hemisphere, which is expected to discover hundreds of pulsars. Here we present early results from LPPS and LOTAS, among which are two independent pulsar discoveries.
\end{abstract}

Keywords. stars: neutron, pulsars: general, surveys

\section{Introduction}

The Low Frequency Array (LOFAR) is a radio telescope under construction in Western Europe (van Haarlem et al. in prep.). LOFAR's core is in the province of Drenthe, The Netherlands. It operates in two bands, a low band $(10-90 \mathrm{MHz})$ and a high band $(110-$ $250 \mathrm{MHz}$ ), each with its own type of antenna. These bands cover the lowest 4 octaves of the radio window. LOFAR is a phased array consisting of many relatively inexpensive antennas, whose signals are digitized and then processed mostly in software. The antennas are grouped in stations where each station is the equivalent of a steerable dish in a traditional interferometer. LOFAR will consist of 40 Dutch stations and 8 international stations (in France, Germany, Sweden and the United Kingdom). All of the core and international stations are complete, and more than half of the remote Dutch stations are also operational. The same software correlator allows LOFAR to operate in both imaging and beam-formed modes. Each beam in the beam-formed mode is synthesized by adding data from multiple stations and acts as the field-of-view of a single-dish telescope. This mode provides the high time resolution required for pulsar survey observations (Stappers et al. 2011). The number of beams that LOFAR can create simultaneously is only constrained by compute power and system throughput, but can be as high as several hundreds, covering up to hundreds of square degrees. In the low band these beams can be pointed anywhere on the sky in the high band they need to be clustered.

Low-frequency observations of pulsars are complicated by 3 effects: dispersion, scattering and higher sky background temperature. Dispersion, the delay caused by free electrons in the interstellar medium (ISM), smears out pulsar signals thus making them less detectable. Since the delay scales with frequency $\nu$ as $\nu^{-2}$, LOFAR observations are strongly affected. Fortunately, the availability of abundant compute power allows incoherent de-dispersion with small channel bandwidths, or even online coherent de-dispersion for up to 40 different trial dispersion measures $\dagger$. Scattering caused by multipath propagation in the clumpy ISM is highly dependent on the line-of-sight, and scales as $\nu^{-4.4}$.

$\dagger$ Intermediate dispersion measure trials can be filled in with offline incoherent de-dispersion. 


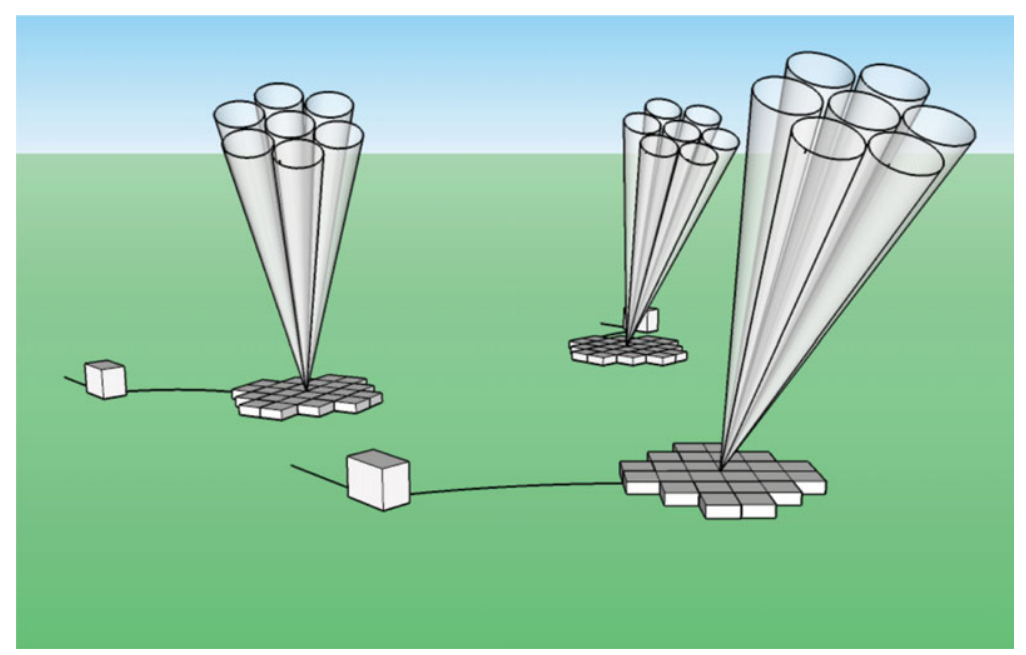

Figure 1. Three LOFAR high-band sub-stations in LPPS mode, where they each create 7 beams of $6.8 \mathrm{MHz}$ bandwidth each. Since each station is pointed in the same direction, LOFAR's central correlator can incoherently combine the station beams. This maintains the large station beam field-of-view, about 75 square degrees for LPPS, whilst increasing the overall sensitivity with square root of the number of substations added. The LPPS survey used up to 44 substations per observation.

It cannot be easily corrected for. This is a problem especially for pulsar observations in the Galactic plane. Conversely, because LOFAR is more strongly affected by scattering, it is an instrument well suited to studying the structure of the ISM with pulsars. Finally, the background sky temperature increases with decreasing observing frequency, as $\nu^{-2.6}$. This effect is mostly a problem towards the Galactic plane. Fortunately, two properties of pulsars potentially increase the chance of a LOFAR detection. Their spectrum generally goes as $\nu^{-1.8}$ and their beams are broader at low frequencies.

LOFAR is an efficient pulsar surveying instrument. LOFAR's antenna elements are sensitive to a large part of the sky, and the correlator can create many beams with a combined field-of-view of tens to hundreds of degrees. Therefore, LOFAR can cover a large part of the sky in little observing time, and/or use long dwell times. The beamforming can happen in two modes: station data can be combined either coherently or incoherently. The coherent mode offers maximum raw sensitivity, while the incoherent mode trades sensitivity for larger field-of-view; see van Leeuwen \& Stappers (2010) for the details of this trade-off. During LOFAR commissioning the LOFAR Pulsar Working Group performed pilot surveys in each of these modes. The first such survey, the LOFAR Pilot Pulsar Survey (LPPS), used incoherent addition of 7 beams created at station level. The second survey, the LOFAR Tied Array Survey (LOTAS), exercised the ability to create 19 tied-array beams by coherently adding station data.

In Section 2 we give an overview of the LPPS survey and present some recent results. In Section 3 we do the same for the LOTAS survey and finally in Section 4 we discuss the lessons learned and the future outlook for pulsar surveys with LOFAR.

\section{The LOFAR Pilot Pulsar Survey}

The LPPS survey was started almost as soon as LOFAR gained the ability to form several beams at station level and combine those beams for all stations at the central correlator. The observations were taken in December 2010 and early January 2011. Each 


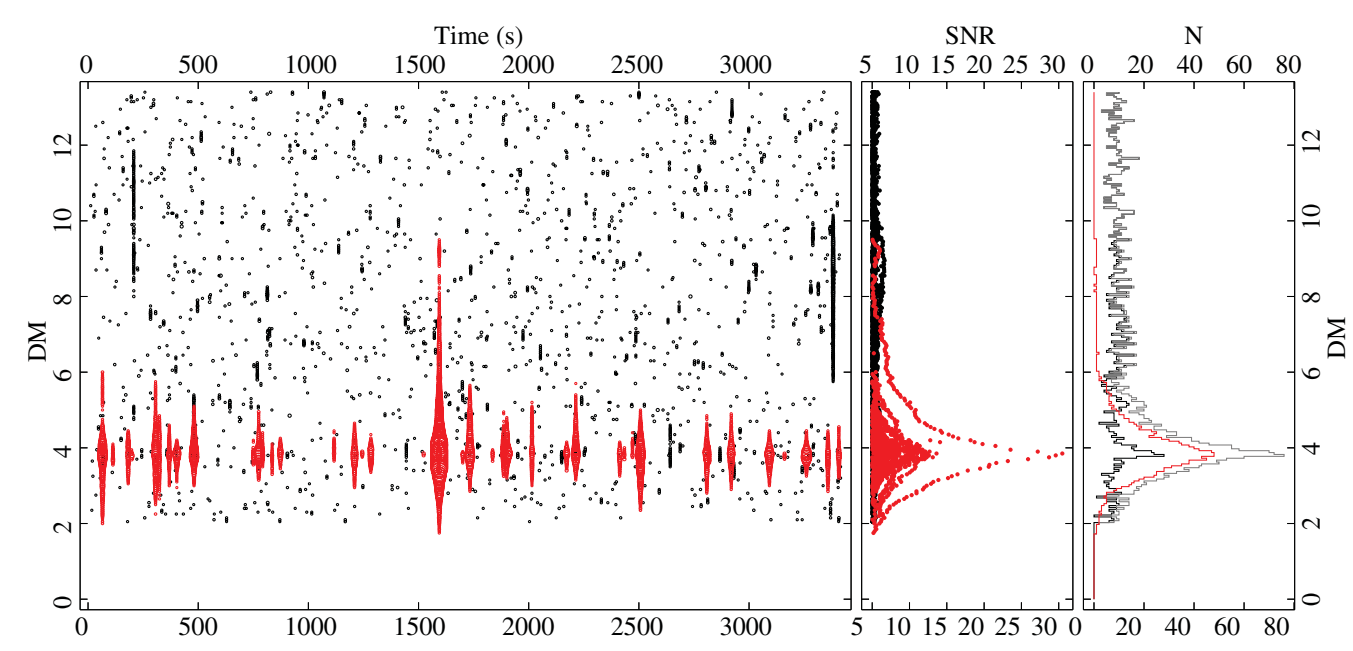

Figure 2. Pulsar PSR J0240+62, a recent low dispersion measure discovery, was re-detected in the LPPS survey. The pulsar, as found by our single pulse search post-processing algorithms, is shown in red and the noise background in black.

LPPS pointing had 7 beams with $6.8 \mathrm{MHz}$ bandwidth, a sampling time of $0.65 \mathrm{~ms}$ and a dwell time of 57 minutes. This long dwell time was possible because each pointing covered about 75 square degrees (see Figure 1). LPPS is comprised of about 250 such observations. The search processing is now complete, and candidate inspection is in progress.

The search was performed with a custom Python pipeline using tools from the PRESTO (Ransom 2001) pulsar data reduction package. The data were reduced at ASTRON and the University of Manchester. We searched the data for both periodicities and single dispersed pulses. In Figure 2 we present an interesting single-pulse detection of PSR J0240+62, a recently discovered pulsar with a low dispersion measure (DM) of $\sim 4$ (Hessels et al. 2008). This shows that LOFAR has the outstanding ability to detect lowDM sources. The periodicity search yielded the first independent $\dagger$ discovery of a pulsar, PSR J2317+68, with LOFAR (see the left panel of Figure 3).

\section{The LOFAR Tied Array Survey}

LOTAS, the second pulsar commissioning survey, was set up to test the ability to create multiple tied-array beams for surveying. For this survey, we used the 6 inner-most stations to create 19 beams, each with LOFAR's full bandwidth of $48 \mathrm{MHz}$. The observations were 17 minutes each with a sampling time of $1.3 \mathrm{~ms}$. Since these 6 stations act as one large, single station the beams cover approximately 3.7 square degrees total, a smaller area than those for the LPPS survey. For LOTAS we observed about 200 pointings.

The data reduction for this survey is being performed with an updated version of the LPPS data reduction pipeline, running at The University of Manchester and at the SARA Grid Node in Amsterdam. The LOTAS survey has so far yielded the second, again independent $\ddagger$, discovery of a pulsar, PSR J2243+69 (see the right panel of Figure 3).

$\dagger$ Only weeks before, the GBNCC pulsar survey had discovered this pulsar (priv. comm.). $\ddagger$ This pulsar has also been discovered recently by the GBNCC survey (priv. comm.). 
$\mathrm{P}=0.813 \mathrm{~s}$
$\mathrm{DM}=71.9 \mathrm{pc} / \mathrm{cm}^{3}$

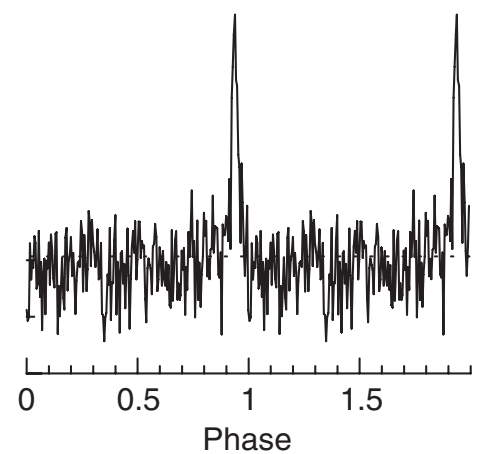

$\mathrm{P}=0.855 \mathrm{~s}$

$\mathrm{DM}=67.8 \mathrm{pc} / \mathrm{cm}^{3}$

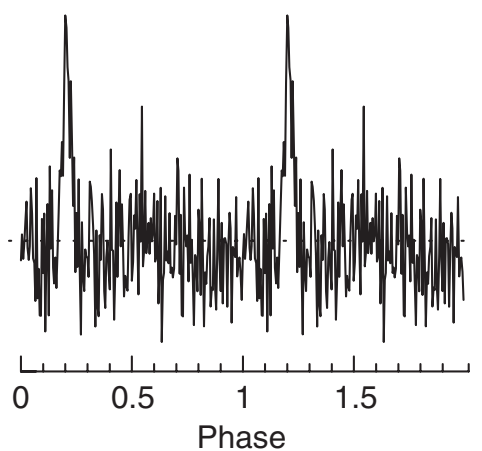

Figure 3. The two independent discoveries in the LOFAR pulsar commissioning surveys. On the left PSR J2317+68, found in the LPPS data; on the right PSR J2243+69, found in LOTAS.

\section{Discussion and further work}

The independent discovery of two pulsars in LOFAR's pulsar commissioning surveys shows that LOFAR is already competitive for such work. It needs to be emphasized that the LPPS survey used LOFAR in a very early stage when it was not yet fully calibrated. The data processing for the LPPS and LOTAS surveys is not yet complete and we expect that these surveys will yield more discoveries soon. For LPPS we are wrapping up the processing and inspecting the results. Because of the large field-of-view $\times$ time on sky we aim to derive a limit on the rate of bright radio bursts, in the absence of strong scattering constraints.

LOFAR's capabilities are still being extended. For targeted observations its sensitivity will increase because the central single clock will be rolled out beyond the inner-most 6 stations, connecting a total of 24 core stations by the end of 2012 - providing a 4 -fold increase in raw sensitivity. Better usage of the available network bandwidth is expected to increase the observing bandwidth to $80 \mathrm{MHz}$. Monitoring of the individual stations' data quality and a better understanding of the interference environment will further increase the data quality compared to that of our early surveys.

Now that LOFAR is emerging from its commissioning period, the LOFAR Pulsar Working Group is gearing up to perform a deeper survey of the northern celestial hemisphere. This survey, the LOFAR Tied Array All-Sky survey (LOTAAS), will use 61 tied-array beams formed with only the 6 inner-most stations. A move to using all core stations would increase raw-sensitivity by a factor 4 over this setup. The decrease in field-of-view, however, cannot be compensated for by creating more beams (as the computing and storage requirements exceed what is available currently or in the near future). With a sampling time of $164 \mu \mathrm{s}$ LOTAAS, unlike LPPS and LOTAS, is designed to be sensitive to both regular and millisecond pulsars.

\section{References}

van Haarlem, M., et al. 2012, A\&SA, in prep.

Hessels, J. W. T., Ransom, S. M., Kaspi, V. M., et al. 2008, American Institute of Physics Conference Series, 983 , pages $613-615$

van Leeuwen, J. \& Stappers, B. W. 2010, A\& A, 509, 7

Ransom, S. M. 2001, PhD thesis, Harvard University

Stappers, B. W., Hessels, J. W. T., Alexov, A., et al. 2011, A\& A, 530, 80 